Auf Wachstumskurs

\title{
Lange Zahlungsziele belasten freiberufliche Pflegekräfte
}

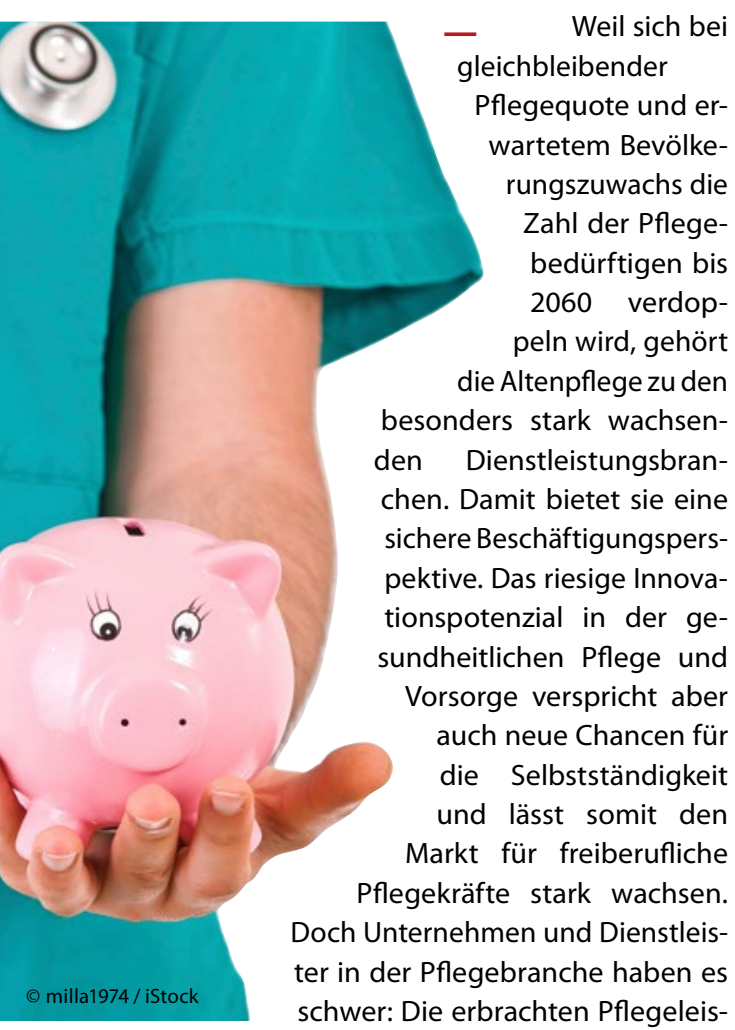

tungen sollen nicht nur immer günstiger, sondern auch mit einem immer späteren Zahlungsziel angeboten werden. Für selbstständige Pflegekräfte, die auf Vorleistung arbeiten, ist das jedoch nahezu unmöglich. Zudem müssen laufende Kosten wie Steuern, Miete, Auto und Versicherungen fortlaufend beglichen werden. Kosten, durch die die Liquidität der Freiberufler gebunden wird.

Um diese wieder freizusetzen, kann das innovative Finanzmodell Factoring helfen. Mit Hilfe von Factoring werden offene Forderungen angekauft und der Rechnungsbetrag innerhalb von 48 Stunden abzüglich einer Servicegebühr an den Freiberufler ausgezahlt. Eine zusätzliche Arbeitserleichterung schafft die Übernahme des kompletten Mahnwesens sowie der Ausfallschutz der Forderung. So kann sich die Pflegekraft wieder ganz darauf konzentrieren, Menschen täglich zu einem einfacheren und besseren Leben zu verhelfen.

www.rechnung48.de

\section{Expertenstandard \\ „Dekubitusprophylaxe in der Pflege"}

— Für die zweite Aktualisierung des Expertenstandards "Dekubitusprophylaxe in der Pflege" unter der wissenschaftlichen Leitung von PD Dr. Jan Kottner, Charité Universitätsmedizin in Berlin, sucht das DNQP Expertinnen und Experten mit pflegewissenschaftlicher und praktischer Expertise. Der Aktualisierungszeitraum wird sich über ca. 18 Monate mit zwei Sitzungen am 20. Juni 2016 und 11. November 2016 erstrecken. Im Rahmen der Aktualisierung sollen erstmalig Indikatoren für das interne Qualitätsmanagement entwickelt werden.

Basierend auf der aktuellen Literaturanalyse wird die Expertenarbeitsgruppe in enger Zusammenarbeit mit dem wissenschaftlichen Team des DNQP eine aktualisierte Version des Expertenstandards und der Kommentierungen erarbeiten, die im Rahmen einer Konsultationsphase mit der Fachöffentlichkeit abgestimmt wird. Anschließend sollen dem Qualitätsniveau des aktualisierten Expertenstandards entsprechende Indikatoren für das einrichtungsinterne Qualitätsmanagement identifiziert und in einem Praxisprojekt erprobt werden. Voraussetzung für die Teilnahme ist der Nachweis einer ausgewiesenen Fachexpertise beispielsweise eine spezielle Fachweiterbildung, wissenschaftliche Qualifikation, Mitwirkung an der Einführung innovativer Praxisprojekte, Fachveröffentlichungen und -vorträge oder Erfahrung in der Arbeit mit Expertenstandards. Neben der fachlichen Expertise werden die Bewerber gebeten, eigene Interessen, Verbindungen zur Industrie oder Interessensverbänden offen zu legen, um so die wissenschaftliche und institutionelle Unabhängigkeit der Expertenstandards garantieren zu können. Bewerbungen bitte bis zum 4. Januar 2016 an: Deutsches Netzwerk für Qualitätsentwicklung in der Pflege (DNQP) an der Hochschule Osnabrück, Prof. Dr. Andreas Büscher, Postfach 19 40, 49009 Osnabrück (dnqp@ hs-osnabrueck.de). 